\title{
Relevance of Helicobacter pylori infection in Egyptian multiple sclerosis patients
}

\author{
Steven Emil Gerges ${ }^{*}$ (D), Taha Kamel Alosh, Salma Hamed Khalil and Mona Mokhtar Waheed El Din
}

\begin{abstract}
Background: Multiple sclerosis (MS) is an autoimmune demyelinating disorder. The etiology of MS remains unknown exactly. Helicobacter pylori heat shock proteins were suggested as a potential trigger of immune system causing MS.

Objectives: The aim of this study was to assess the level of anti-Helicobacter pylori heat shock proteins 60 (Hp hsp60) antibodies at patients of MS and to correlate it with various epidemiological and clinical data.

Subjects and methods: This study design was a cross-sectional case control one. A total of 65 patients with multiple sclerosis diagnosed according to 2010 revised McDonald criteria and other 65 age- and sex-matched healthy controls were included in this study. All participants were subjected to full history taking, complete neurological examination including Expanded Disability Status Scale (EDSS) for the patients, measurement of serum level of anti-Hp hsp60 lgG using ELISA technique, and MRI brain for all the patients, being a goldstone for inclusion in the study.

Results: There was statistically significant high level of anti-Hp hsp60 lgG at MS patients especially secondary progressive multiple sclerosis (SPMS) patients. Moreover, a positive statistically significant correlation was found between it and age of patients, duration of illness, and EDSS.
\end{abstract}

Conclusion: We conclude that hsp60 of Hp may be a useful biomarker for attesting course progression in MS.

Keywords: Antibodies, Autoimmune, Heat shock protein 60, Helicobacter pylori, Multiple sclerosis

\section{Introduction}

The exact prevalence of multiple sclerosis in Egypt cannot be given since no organized survey for this purpose was carried out and door to door study was difficult. A community-based survey using 2005 revised McDonald's criteria for diagnosis of multiple sclerosis (MS) in $\mathrm{Al}$ Quseir, Egypt, has found an MS prevalence of 13.74/ $100,000[1]$.

There is also data scarcity on the prevalence of Helicobacter pylori infection in an Egyptian population and the only study found was a study in a rural area of the country that revealed an overall seropositivity rate of $91.7 \%$ of this population. The rate of infection was different in different age groups with an increasing trend in older ages [2].

The etiology of MS remains unknown. Several environmental factors, including microbial agents, have been considered potential inducers of the disease [3].

\footnotetext{
* Correspondence: Stevnemil7@gmail.com

Faculty of Medicine, Ain Shams University, 19 Awad Fahmy st-El Zatoun, Cairo, Egypt
}

Among the microbial agents, Helicobacter pylori $(\mathrm{Hp})$ has been considered a possible infectious trigger of the disease [4]. This assumption may be supported by high incidence and prevalence of gastrointestinal symptoms at MS [5].

At the antigen level, several $\mathrm{Hp}$ antigens have been considered important for the loss of immunological tolerance to myelin antigens, particularly heat shock proteins (hsp) [6].

High degree of sequence homology between mammalian and pathogenic heat shock protein has been found in several studies. The immune system may recognize heat shock proteins as dominant pathogenic antigens or potentially harmful self-antigens due to its high conserved nature. Conserved epitopes of heat shock proteins among mammalian cells and prokaryotes may lead to cross reactivity and induces immune reactivity to self-heat shock proteins which eventually results in autoimmune diseases [7].

The immune reaction that occurs in MS is thought to trigger a process of neurodegenerative damage that leads 
to clinical signs. In this scenario, extracellular hsp, namely hsp60 and hsp70, exacerbates the immune response by acting as an adjuvant for myelin peptides and as a proinflammatory cytokines [8].

In the past, high levels IgG antibodies against hsp70 have been reported in the cerebrospinal fluid (CSF) of patients with MS. However, significant difference in the levels of antibodies against hsp27, hsp60, or hsp90 was not observed [9].

Antibody responses to Hp-specific hsp60 have not been studied in great detail in MS despite being one of the most immunogenic heat shock proteins [10].

In the present study, we assess the level of this antibody in patients with MS as a biomarker for the inflammatory processes and for possible etiological role.

\section{Subjects and methods}

A group of 65 patients with MS from the outpatient MS clinic of Ain Shams University hospital chosen randomly and a group of 65 age- and sex-matched healthy controls were included in this study. The study was performed between July 2016 and July 2017. The procedures followed were in accordance with the ethical standards of the responsible committee on human experimentation and with the principles of Helsinki Declaration [11]. Informed consent was obtained from all participants, and ethical committee permission from our institution was obtained before starting our work. Inclusion criteria were as follows: patients known to have MS according to revised 2010 McDonald criteria for diagnosis of MS [12]. Exclusion criteria were as follows: patients with neuromylitis optica (Devic's disease) or other demyelinating and inflammatory disorders of central nervous system (CNS), and other chronic medical disorders (diabetes mellitus, chronic kidney disease, chronic liver disease, other endocrinal disorders).

All the participants were subjected to the following: clinical evaluation including general medical history and examination, full neurological history and examination, diagnosis of definite MS according to revised 2010 McDonald criteria for diagnosis of MS [12], assessment of severity of MS in each patient by using Expanded Disability Status Scale (EDSS) [13], and quantitative assessment of the level of IgG antibodies against Hp hsp60 at the serum of both cases and controls by using enzymelinked immunosorbent assay (ELISA) technique; the detection range of the kit was from $(78.13-5000 \mathrm{pg} / \mathrm{mL}$ ) and the materials and instruments were obtained from Elabscience biotechnology (www.elabscience.com). Magnetic resonance imaging (MRI) brain (all cases were subjected to MRI brain with T1 weighted with and without contrast, T2 weighted and fluid-attenuated inversion recovery (Flair) scans to apply proposed MAGNIMS criteria for diagnosis of MS [14]).

\section{Statistical analysis}

Statistical presentation and analysis of the present study was conducted, using the mean, standard deviation, student $t$ test, Chi-square, linear correlation coefficient, and analysis of variance (ANOVA) tests. Collected data in this study were analyzed using the statistical package for the social sciences (SPSS, version 17; SPSS Inc., Chicago, Illinois, USA).

Unpaired Student $T$ test was used to compare between two groups in quantitative data. Chi-square test was used to compare independent row and column variables without indicating strength or direction of the relationship. Fisher's exact test and Yates' corrected chi-square are computed for analysis of contingency tables. Linear correlation coefficient was used for detection of correlation between two quantitative variables in one group. ANOVA test was used for comparison among different times in the same group in quantitative data.

\section{Results}

The study included 65 patients with definite multiple sclerosis and 65 age- and sex-matched healthy individuals. The case group included 17 males (26.15\%) and 48 females $(73.85 \%)$ and their age ranged from 15 to 60 years with mean age of $34.723 \pm 9.711$ years. Forty-two patients $(64.2 \%)$ were diagnosed as relapsing remitting multiple sclerosis (RRMS), 22 patients (33.85\%) were diagnosed as secondary progressive multiple sclerosis (SPMS), and 1 patient $(1.54 \%)$ was diagnosed as primary progressive multiple sclerosis (PPMS). The age of onset of multiple sclerosis among the cases ranged from 13 to 49 years old with the mean age of onset was $26.908 \pm 7.814$ years. The duration of MS among the cases ranged from 1 to 28 years with the mean duration was 14.5 years. The EDSS ranged from 2 to 7 while the median EDSS was 4.5. The control group included 20 males (30.77\%) and 45 females (69.23\%) and their age ranged from 18 to 38 years with mean age of $35.215 \pm 9.672$ years. These data are presented in Table 1.

In our study, we found a high statistically significant difference in level of Hp hsp60 IgG in cases compared to controls $(P=0.001)$. There was no significant statistical difference between level of anti-Hp hsp60 IgG at male and female patients of MS $(P=0.646)$. As regard the course of the MS, patients with SPMS were found to have statistically significant higher levels of anti $\mathrm{Hp}$ hsp60 IgG compared to those with RRMS course $(P=$ 0.001). These data are presented in Table 2 .

Correlation between age of MS patients and the level of anti-Hp hsp60 IgG showed that there is a statistically significant correlation between them $(P=0.008)$ (Fig. 1). A statistically significant correlation was also found between anti-Hp hsp60 IgG and duration of MS $(P=$ 0.012) (Fig. 2). However, in both previous figures, the 
Table 1 Descriptive statistics of the study

\begin{tabular}{|c|c|c|c|c|c|c|c|c|}
\hline \multirow[t]{3}{*}{ Gender } & \multicolumn{6}{|c|}{ Groups } & \multicolumn{2}{|c|}{ Chi-square } \\
\hline & \multicolumn{2}{|c|}{ Case } & \multicolumn{2}{|c|}{ Control } & \multicolumn{2}{|l|}{ Total } & \multirow[b]{2}{*}{$x^{2}$} & \multirow[b]{2}{*}{$P$ value } \\
\hline & $N$ & $\%$ & N & $\%$ & $N$ & $\%$ & & \\
\hline Male & 17 & 26.15 & 20 & 30.77 & 37 & 28.46 & 0.340 & 0.560 \\
\hline Female & 48 & 73.85 & 45 & 69.23 & 93 & 71.54 & & \\
\hline Total & 65 & 100.00 & 65 & 100.00 & 130 & 100.00 & & \\
\hline \multirow[t]{2}{*}{ Age } & \multicolumn{6}{|c|}{ Groups } & \multicolumn{2}{|l|}{$T$ test } \\
\hline & \multicolumn{3}{|c|}{ Case } & \multicolumn{3}{|l|}{ Control } & $T$ & $P$ value \\
\hline Range & \multicolumn{3}{|c|}{$18-60$} & \multicolumn{3}{|l|}{$15-60$} & -0.290 & 0.773 \\
\hline Mean \pm SD & \multicolumn{3}{|c|}{$34.723 \pm 9.711$} & \multicolumn{3}{|c|}{$35.215 \pm 9.672$} & & \\
\hline \multicolumn{9}{|c|}{ Course of MS } \\
\hline & & & \multicolumn{2}{|l|}{ N } & \multicolumn{2}{|l|}{$\%$} & & \\
\hline \multicolumn{3}{|l|}{ RRMS } & \multicolumn{2}{|l|}{42} & \multicolumn{2}{|l|}{64.62} & & \\
\hline \multicolumn{3}{|l|}{ SPMS } & \multicolumn{2}{|l|}{22} & \multicolumn{2}{|l|}{33.85} & & \\
\hline \multicolumn{3}{|l|}{ PPMS } & \multicolumn{2}{|l|}{1} & \multicolumn{2}{|l|}{1.54} & & \\
\hline \multirow{2}{*}{\multicolumn{3}{|c|}{ Total }} & \multicolumn{2}{|l|}{65} & \multicolumn{2}{|c|}{100.00} & & \\
\hline & & & \multicolumn{2}{|c|}{ Range } & \multicolumn{2}{|c|}{ Mean \pm SD } & & \\
\hline Age of MS & onset & & $13-4$ & & 26.90 & $8 \pm 7.814$ & & \\
\hline Duration of & MS & & $1-28$ & & 14.5 & & & \\
\hline & & & Rang & & Media & & & \\
\hline EDSS & & & $2-7$ & & 4.5 & & & \\
\hline
\end{tabular}

$S D$ standard deviation, RRMS relapsing remitting multiple, SPMS secondary progressive multiple sclerosis, PPMS primary progressive multiple sclerosis, EDSS expanded disability status scale

correlation coefficient $(r)$ was 0.3 which means moderate positive relationship, even being statistically significant. This means that the high level of anti-hsp60 IgG is moderately positively correlated with the age of MS patients and duration of MS. A statistically significant strong positive correlation was found between the level of anti-hsp60 IgG and scores of EDSS ( $P=0.001)$ (Fig. 3).

\section{Discussion}

Antibody responses to Hp-specific hsp60 has not been studied in great detail in MS except at one study by Efthymiou and colleagues [15] that suggested a possible relation between infection by $H$. pylori species containing hsp60 antigens and triggering of MS.

These data encouraged the design of this study in order to investigate such relation and to correlate the results with various epidemiological and clinical data.

In this study, there was a significant higher level of anti $\mathrm{Hp}$ hsp60 IgG at MS patients compared with healthy controls, the level was especially high at SPMS patients suggesting a possible relation between infection by $\mathrm{Hp}$ species containing this $\mathrm{Ag}$ and pathogenesis of MS especially SPMS phenotype. Similar results were found by Efthymiou and colleagues [15]. This high level of reactivity of anti-Hp hsp60 at SPMS may support the recent evidence that $B$ cells and antibodies play an important role at pathogenesis of progressive forms of MS and that compartmentalized CNS inflammation is one of the driving processes behind neurodegeneration and progression at MS; such theory is consolidated by identification of meningeal ectopic B cell follicles in SPMS patients and by successful use of B cell depleting therapy in PPMS patients [16]. Such findings may be also related to the genotype of different populations and how it affects the predisposition to MS and reaction to different antigens; a study by Atya and colleagues showed that interleukin $17 \mathrm{~F}$ (IL-17 F) CT genotype and C allele may be associated with a susceptibility to MS in Egyptian population by a gender-dependent mechanism that contributes to unique predisposition in females [17]. Another Egyptian study showed that signal transducer and activator of transcription 4 (STAT4) polymorphism was significantly associated with multiple sclerosis in Egyptian population [18]. Such relation between genotype

Table 2 Comparison between cases and controls as regard level of anti-Hp hsp60 lgG and its distribution at gender and course of MS patients

\begin{tabular}{|c|c|c|c|c|c|}
\hline \multirow[t]{2}{*}{ Anti hsp60 lgG level (pg/ml) } & \multicolumn{3}{|l|}{ Groups } & \multicolumn{2}{|l|}{$T$ test } \\
\hline & Case & \multicolumn{2}{|l|}{ Control } & $t$ & $P$ value \\
\hline Range & $1150-5000$ & \multicolumn{2}{|l|}{$550-5000$} & 3.383 & $0.001^{*}$ \\
\hline Mean \pm SD & $3421.538 \pm 1385.999$ & \multicolumn{4}{|c|}{$2570.846 \pm 1479.420$} \\
\hline \multirow[t]{2}{*}{ Cases } & & \multicolumn{2}{|c|}{ Anti-hsp60 lgG level (pg/ml) } & \multicolumn{2}{|c|}{$T$ test or ANOVA } \\
\hline & & $N$ & Mean \pm SD & $T$ or $F$ & $P$ value \\
\hline \multirow[t]{2}{*}{ Gender } & Male & 17 & $3555.882 \pm 1416.160$ & \multirow[t]{2}{*}{0.462} & \multirow[t]{2}{*}{0.646} \\
\hline & Female & 48 & $3373.958 \pm 1387.175$ & & \\
\hline \multirow[t]{3}{*}{ Course of MS } & RRMS & 42 & $2775.000 \pm 1186.098$ & \multirow[t]{3}{*}{24.836} & \multirow[t]{3}{*}{$<0.001^{*}$} \\
\hline & SPMS & 22 & $4702.273 \pm 709.708$ & & \\
\hline & PPMS & 1 & $2400.000 \pm 0.000$ & & \\
\hline
\end{tabular}

hsp60 heat shock protein 60, lgG immunoglobulin G, SD standard deviation, ANOVA analysis of variance, RRMS relapsing remitting multiple sclerosis, SPMS secondary progressive multiple sclerosis, PPMS primary progressive multiple sclerosis 


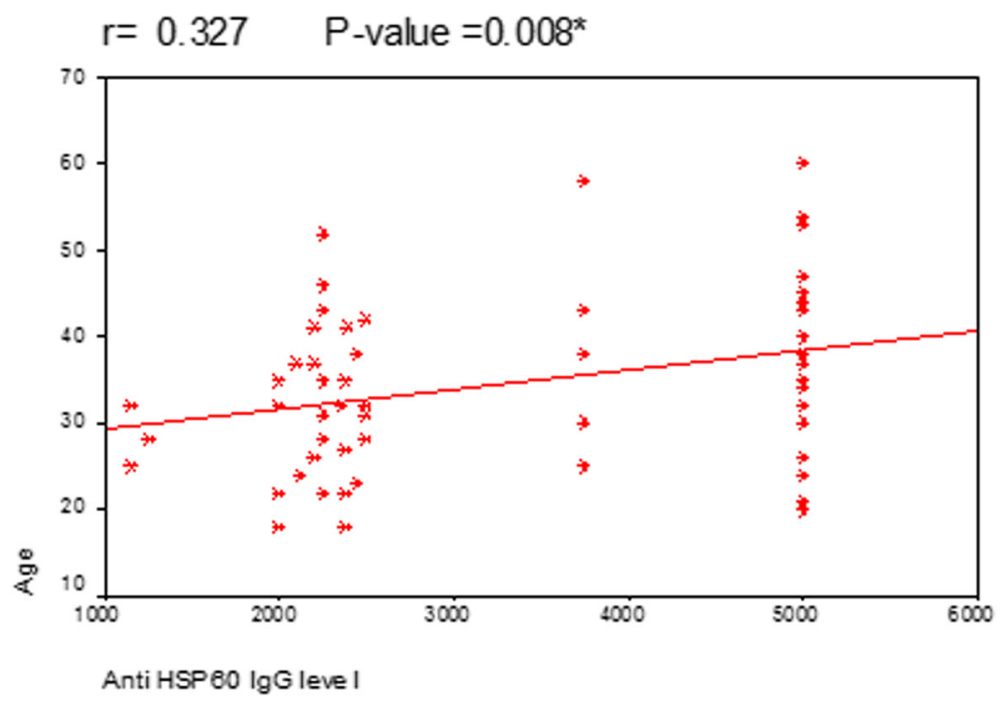

Fig. 1 Correlation between anti Hp hsp60 lgG level and age of the MS patients

and serum level of various antibodies need to be further studied.

A recent study by Lechner and collegues found a significant higher level of Hsp70 at serum of patients with RRMS and CIS than patients with SPMS and claimed that it can be used as a marker of inflammation at MS [19]. This difference in results may be related to the difference in nature between the two types of heat shock proteins or the difference between antigens and antibodies.

In this study, there was also a significant correlation between age of the MS patients, duration of the illness, and EDSS of the patients on one the hand and the level of anti-Hp hsp60 IgG on the other hand but it was not of high statistical significance. Similar results were also found by Efthymiou and colleagues [15] for age of the patients and duration of illness but not for EDSS. Such findings may reflect the tendency of longstanding inflammation to elicit antibody responses to an expanded range of targets [20] but whether this is just an epiphenomenon or has a real contributing role at disease progression is a question need to be further assessed. Lechner and colleagues found no relation between Hsp70 level and age of MS patients [19].

In this study, no significant relation was found between gender of MS patients or the age of onset of the illness and the titer of anti-Hp hsp60 IgG; Efthymiou and colleagues

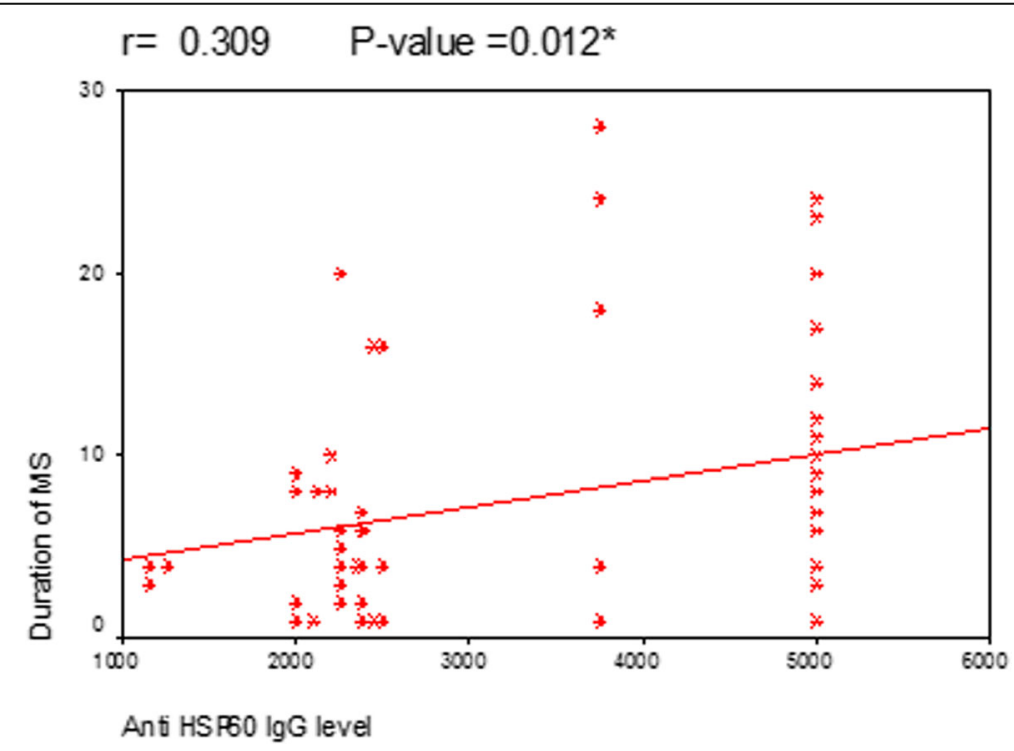

Fig. 2 Correlation between anti Hp hsp60 lgG level and duration of MS 


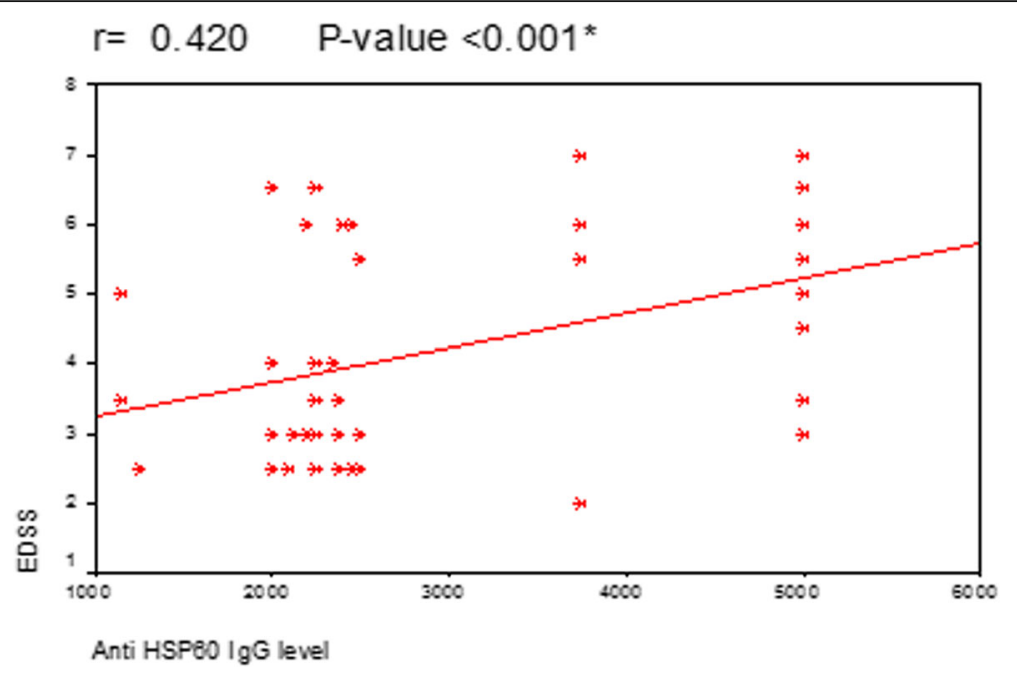

Fig. 3 Correlation between anti Hp hsp60 lgG level and EDSS of MS patients

[15] found a positive correlation with the age of onset but not for the gender, also Lechner and collegues found no relation between gender and Hsp70 level at MS patients [19].

Limitations of this study include the relatively small number of participants especially of SPMS patients, no correlation between gastrointestinal (GIT) symptoms and results. Also, no correlation of the results with the degree of patients' compliance with MS treatments. Moreover, the high financial cost of laboratory analysis of such antibodies at MS patients may be a barrier to its actual application as a marker for progression at disease.

\section{Conclusion}

We conclude from this study that high anti Hp hsp60 IgG level is correlated to MS patients especially SPMS. It can be used as a biomarker for progression of such disease, being significantly correlated to age and duration of illness in a moderate level and EDSS scores to a strong level.

\section{Recommendations}

There is a need for this study to be conducted with a larger number of participants, the relation between heat shock proteins of various species and pathogenesis of MS need to be further studied; there is a need for further studies investigating the role of $\mathrm{Hp}$ hsp60 specific $\mathrm{T}$ cell immune responses in MS patients which could clarify whether their presence in sera of MS patients is just an epiphenomenon or they play a real role at induction of the disease at genetically susceptible individuals; there is a need for further studies assessing the level of this antibody at sera of MS patients at relapse to detect if they play a role at disease exacerbation or not, and correlation between GIT symptoms and level of this antibody also needs to be studied. Also, the degree of compliance with MS medications and how it affects the level of those antibodies need to be further researched.

\section{Abbreviations}

CNS: Central nervous system; CSF: Cerebrospinal fluid; EDSS: Expanded Disability Status Scale; ELISA: Enzyme-linked immunosorbent assay; Et al: and others; FLAIR: Fluid attenuated inversion recovery; GIT: Gastrointestinal; Hp: Helicobacter pylori; HSPs: Heat shock proteins; Ig: Immuno globulin; IL: Interleukin; MAGNIMUS: Magnetic resonance imaging in MS;

MRI: Magnetic resonance imaging; MS: Multiple sclerosis; OD: Optical density; PG: Picogram; PPMS: Primary progressive multiple sclerosis; RRMS: Relapsing remitting multiple sclerosis; SPMS: Secondary progressive multiple sclerosis; STAT4: Signal transducer and activator of transcription 4

\section{Acknowledgements \\ Not applicable.}

Funding

Self-financing.

Availability of data and materials

All data and materials that support findings in this manuscript are available. Please contact the author for data requests.

\section{Authors' contributions}

SE carried out the immunological studies and statistical analysis, MM participated in the sequence alignment and drafted the manuscript, TA and $\mathrm{SH}$ conceived of the study and participated in its design and coordination. All authors read and approved the final manuscript.

\section{Ethics approval and consent to participate}

The procedures followed were in accordance with the ethical standards of the responsible committee on human experimentation and with the principles of Helsinki Declaration. Informed consent was obtained from all participants and from patients' parents in the case of minors, and ethical committee permission from Faculty of Medicine Ain Shams university ethical committee at 24/9/2017.

\section{Consent for publication}

Not applicable.

\section{Competing interests}

The authors declare that they have no competing interests.

\section{Publisher's Note}

Springer Nature remains neutral with regard to jurisdictional claims in published maps and institutional affiliations. 
Received: 27 April 2018 Accepted: 18 November 2018

Published online: 07 December 2018

\section{References}

1. El-Tallawy HN, Farghaly WMA, Badry R, Metwally NA, Shehata GA, Rageh TA, et al. Prevalence of multiple sclerosis in Al Quseir city, Red Sea governorate, Egypt. Neuropsychiatr Dis Treat. 2016;12:155-8.

2. El Dine SS, Mubarak M, Salama R, et al. Low seroprevalence of anti-CagA antibodies in spite of high seroprevalence of anti-H pylori antibodies in rural Egyptian community. Res J Med Med Sci. 2008;3:118-23.

3. Grigoriadis N, Hadjigeorgiou GM. Virus-mediated autoimmunity in multiple sclerosis. J Autoimmun. 2006;3:1.

4. Smyk DS, Koutsoumpas AL, Mytilinaiou MG, Rigopoulou El, Sakkas LI, Bogdanos DP. Helicobacter pylori and autoimmune disease: cause or bystander. J Gastroenterol. 2014:20:613-29.

5. Levinthal DJ, Rahman A, Nusrat S, O'Leary M, Heyman R, Bielefeldt K. Adding to the burden: gastrointestinal symptoms and syndromes in multiple sclerosis. Mult Scler Int. 2013;2013:319201.

6. Scotti C, Mignosi P, Filipazzi P, Lazze MC, Savio M, Cappelletti D, et al. Molecular alterations in fibroblasts exposed to helicobacter pylori broth culture filtrate: a potential trigger of autoimmunity? Helicobacter. 2010;15:76-7.

7. Grundtman C, Kreutmayer SB, Almanzar G, Wick MC, Wick G. Heat shock protein 60 and immune inflammatory responses in atherosclerosis. Arterioscler Thromb Vasc Biol. https://doi.org/10.1161/ATVBAHA.110.217877 Author manuscript; available in PMC 2011 Nov 10

8. Fleshner M, Johnson JD. Endogenous extra-cellular heat shock protein 72 : releasing signal(s) and function. Int J Hyperth. 2005;21(5):457-71.

9. Chiba S, Yokota S, Yonekura K, Tanaka S, Furuyama H, Kubota H, et al. Autoantibodies against HSP70 family proteins were detected in the cerebrospinal fluid from patients with multiple sclerosis. J Neurol Sci. 2006; 241(1-2):39-43.

10. Gonzalez-Lopez MA, Velazquez-Guadarrama N, Romero-Espejel ME, OlivaresTrejo JDE. Helicobacter pylori secretes the chaperonin GroEL (HSP60), which binds iron. FEBS Lett. 2013;587:1823-8.

11. World Medical Association. 1964. Declaration of Helsinki.

12. Polman $\mathrm{CH}$, Reingold SC, Banwell B, Clanet M, Cohen JA, Filippi M, et al. Diagnostic criteria for multiple sclerosis: 2010 revisions to the McDonald criteria. Ann Neurol. 2011;69(2):292-302.

13. Kurtzke JF. Rating neurologic impairment in multiple sclerosis: an expanded disability status scale (EDSS). Neurology. 1983;33(11):1444-52.

14. Montalban X, Tintoré M, Swanton J, Barkhof, Fazekas F, Filippi M, et al. MRI criteria for MS in patients with clinically isolated syndromes. Neurology. 2010;74(5):427-34.

15. Efthymiou G, Dardiotis E, Liaskos C, Marou E, Tsimourtou V, Scheper T, Meyer W, et al. Anti-hsp60 antibody responses based on helicobacter pylori in patients with multiple sclerosis: (ir)relevance to disease pathogenesis. J Neuroimmunol. 2016;298:19-23.

16. Frausen J, de Bock L, Somers V. B cells and antibodies in progressive multiple sclerosis: contribution to neurodegeneration and progession. Autoimmun Rev. 2016;15(9):896-9.

17. Atya HB, Ali SA, Hegazy MI, et al. Is rs763780 in IL-17F gene considered risk factor to multiple sclerosis in Egyptian patients? Meta Gene. 2017;14:124-8.

18. Nageeb RS, Omran AA, Nageeb GS, Yousef MA, Mohammad YAA, Fawzy A STAT4 gene polymorphism in two major autoimmune diseases (multiple sclerosis and juvenile onset systemic lupus erythematosus) and its relation to disease severity. Egypt J Neurol Psychiatr Neurosurg. 2018;54:16.

19. Lechner P, Buck D, Sick L, Hemmer B, Multhoff G. Serum heat shock protein 70 levels as a biomarker for inflammatory processes in multiple sclerosis. Mult Scler J Exp Transl Clin. 2018;4(2):2055217318767192.

20. Mienel E, Derfuss T, Krumbholz M, Probstel AK, Hohelfeld R. Humoral autoimmunity in multiple sclerosis. Neurol Sci. 2011;306(1-2):180-2.

\section{Submit your manuscript to a SpringerOpen ${ }^{\circ}$ journal and benefit from:}

- Convenient online submission

- Rigorous peer review

- Open access: articles freely available online

- High visibility within the field

- Retaining the copyright to your article

Submit your next manuscript at $\boldsymbol{\nabla}$ springeropen.com 